\title{
Aprendizagem Baseada em Projetos no Curso de Engenharia Civil: na Visão de Diferentes Instituições, Docentes e Acadêmicos
}

\author{
Project-Based Learning in the Civil Engineering Course: View of Different \\ Institutions, Teachers and Academics
}

\author{
Mara Cristina de Almeida ${ }^{1}$, Silvia Sonia da Silva ${ }^{2}$, Scarlet Karina Montilla Barrios ${ }^{3}$, Vitor Hugo \\ Oligini Wessling $^{4}$ e João Pedro de Araújo Nogueira ${ }^{5}$
}

1. Engenheira de pesca. Licenciatura em matemática. Mestre em Zootecnia. Doutora em Aquicultura pela UNESP/CAUNESP. Docente da Faculdade de IPORÃ e da Secretaria do Estado do Paraná - SEED/PR.

2. Engenheira civil pela Universidade Estadual de Maringá. Mestre em Engenharia Hidráulica e Saneamento pela USP. Doutoranda em Energia e Sustentabilidade pela UNILA.

3. Engenheira civil pela Universidad de los Andes Venezuela. Mestre em Engenharia Estrutural pela Universidad de los Andes. Professora na Universidade Nacional Experimental e Politécnica das Forças Armadas. Pesquisadora do Centro de Vivienda y Habitad Venezuela.

4. Engenheiro Civil pelo Centro Universitário Dinâmica das Cataratas. Especialista de Estruturas de Concreto e Fundações no Instituto Brasileiro de Educação Continuada - INBEC. Especialista em Metodologias Ativas pelo Centro Universitário União das Américas. Formação em MBA em Plataforma BIM no Instituto Brasileiro de Educação Continuada - INBEC. Atualmente sócio-administrador de escritório de engenharia. Componente do corpo técnico no Parque Tecnológico Itaipu.

5. Engenheiro civil pelo Centro Universitário Dinâmica das Cataratas. Especialista em estrutura de concreto armado e fundações pela Universidade Paranaense em Cascavel, PR.

mara_macrisal@hotmail.com

Palavras-chave

Aprendizagem na engenharia

Metodologia ativa

Pesquisa educacional

\section{Keywords}

Active methodology

Educational research

Engineering learning

Artigo recebido em: 20.01.2020

Aprovado para publicação em:

16.10.2020
Resumo: A metodologia de Aprendizagem Baseada em Projeto aplicada ao ensino no curso de engenharia civil, além de multidisciplinar permite ao acadêmico agregar conhecimentos e diferentes habilidades por meio de um processo de análise de diferentes questões que vão desde as mais simples as complexas, de modo que o aprendizado seja mais eficiente. E ainda permite os mesmos aprendem de forma mais práticas diferentes ramos e disciplinas do curso. Deste modo este trabalho pretendeu apresentar a visão de diferentes ins tituições e grupos relacionados ao ensino sobre utilização de projetos no processo de ensino. Foram realizadas pesquisas de campo através de um questionário online entre os dias 03/09/18 há 21/09/2018 paralelamente em diferentes instituições de ensino, entre docentes e acadêmicos sobre a metodologia em questão. Após realização da pesquisa os dados foram tabulados no programa em tabelas no Microsoft Excel (2010) e submetidos a um teste de médias (Tukey). Os resultados demonstram que $88 \%$ dos entrevistados são acadêmicos e $12 \%$ docentes, $61,9 \%$ estudam ou trabalham em instituições de ensino privada. Com relação ao método, $74 \%$ já utilizaram projetos e $100 \%$ dos entrevistados consideram importante a aplicabilidade dos projetos no curso. Os entrevistados atribuem o conceito de muito bom para a metodologia $(52,4 \%)$, mas como pontos negativos consideram que a falta interesse e dedicação dos grupos envolvidos $(38,1 \%)$ influência e a falta de efetividade dos projetos na prática. Assim podemos evidenciar que a utilização de projetos nos cursos de engenharia civil demonstram serem eficientes, mas precisam ser mais práticos e atrativos para diferentes grupos. 


\begin{abstract}
The Project-Based Learning methodology applied to teaching in the civil engineering course, in addition to being multidisciplinary, allows the academic to aggregate knowledge and different skills through a process of analysis of different questions, ranging from the simplest to the complex ones, so that the learning is more efficient. And it also allows them to learn in a more practical way different branches and disciplines of the course. In this way, this work intended to present the vision of different institutions and groups related to teaching about the use of projects in the teaching process. Field research was carried out through an online questionnaire between 9/3/18 and 9/21/2018 in parallel at different educational institutions, including teachers and academics about the methodology in question. After conducting the research, the data were tabulated in the program in tables in Microsoft Excel (2010) and subjected to a test of means (Tukey). The results show that $88 \%$ of respondents are academics and $12 \%$ teachers, $61.9 \%$ study or work in private education institutions. Regarding the method, $74 \%$ have already used projects and $100 \%$ of the interviewees consider the applicability of the projects in the course to be important. The interviewees attribute the concept of very good to the methodology (52.4\%), but as negative points they consider that the lack of interest and dedication of the groups involved (38.1\%) influence and the lack of effectiveness of the projects in practice. Thus we can evidence that the use of projects in civil engineering courses proves to be efficient, but they need to be more practical and attractive to different groups.
\end{abstract}

\title{
INTRODUÇÃO
}

A formação profissional propõe que o estudante possa desenvolver e praticar, em sala de aula, a maior quantidade possível de atividades que demonstrem a realidade da profissão, para a qual está se preparando. Na relação entre a sala de aula e a prática profissional, é essencial que se construa um ambiente de aprendizagem em que o estudante se sinta motivado a aprender, participante do processo e, acima de tudo, que perceba o real valor do conteúdo que está sendo abordado. O processo de ensino e aprendizagem precisa evoluir ao mesmo tempo em que as ferramentas tecnológicas se tornam componentes quase que obrigatórios à sobrevivência do profissional moderno (SANTIN \& AHLERT, 2017).

Grandes transformações são observadas em todas as áreas da atividade humana e uma das áreas particu larmente afetada por essas mudanças é a Engenharia, pois a revolução tecnológica é um dos fatores que contribui para isso, tendo como consequência a demanda por engenheiros mais flexíveis e com qualificações que extrapolam a especialização técnica. Assim, o ponto crucial para os cursos de Engenharia é a eficiência do aprendizado, que exige mudanças metodológicas qualitativas para o acompanhamento dessas transformações (MASSON et al., 2012).

Segundo Cipolla (2016), aprendizagem baseada em projetos, permite a diferentes grupos de estudantes confrontem questões e problemas do mundo reais significativos para eles, determinando assim a maneira mais prática de abordá-los e estabeleçam uma ação cooperativa em busca de soluções. Este ainda salienta que este método de aprendizagem consegue acomodar mudanças e atualizações tecnológicas que estão em andamento, servindo de interface para que os estudantes desenvolvam as habilidades nessas inovações que se dão constantemente. A metodologia de Aprendizagem Baseada em Projeto (Project-Based Learning - PBL) aplicada ao ensino no curso de engenharia civil, além de multidisciplinar permite ao acadêmico agregar co nhecimentos e diferentes habilidades por meio de um processo de análise de diferentes questões que vão desde as mais simples as complexas, de modo que o aprendizado seja mais eficiente. E ainda os mesmos podem aprendem de forma mais práticas diferentes ramos e disciplina da engenharia civil.

As características relacionadas a Aprendizagem Baseada em Projetos deve incluir conteúdos teóricos, condições de desenvolvimento, atividades e resultados, para resolução de problemas do cotidiano. E ainda possibilitar aos estudantes autonomias para organizarem os seus conteúdos e serem responsáveis pela cons trução do seu saber, facilitando o entendimento e retenção da informação.

ALMEIDA, M.C. et al. Aprendizagem Baseada em Projetos no Curso de Engenharia Civil: na Visão de Diferentes Instituições, Docentes e Acadêmicos.
Pleiade, 14(30): 71-78, Jan.-Jun., 2020

DOI: 10.32915/pleiade.v14i30.667 
Araújo (2008), Toyohara et al. (2010) constataram que o trabalho por meio de projetos como uma estratégia para construção dos conhecimentos, entendendo estratégia como uma ação que pressupõe decisões, es colhas, apostas, riscos e incertezas. E ainda os autores, afiram que a metodologia citada é diferente de um programa de conteúdos, que é previsto e seguido na sua íntegra, o qual dificulta a abertura para as novidades que surgem durante o seu desenvolvimento, “engessando” a ação docente e impedindo a participação mais ativa dos estudantes na construção do conhecimento.

Em conforme com essas novas metodologias de aprendizado este artigo pretende apresentar as diferentes visões sobre aprendizagem baseada em projetos aplicada aos cursos de engenharia, especificamente ao curso de engenharia civil e ainda enfatizar os principais aspectos sobre o tema em questão.

\section{METODOLOGiA}

Para este trabalho foram realizadas pesquisas com questionário online através da ferramenta do google para pesquisas com perguntas relacionadas a aprendizagem baseada em projetos no curso de engenharia civil

Tabela 1. Questionário sobre aprendizagem baseada em projetos no curso de engenharia civil.

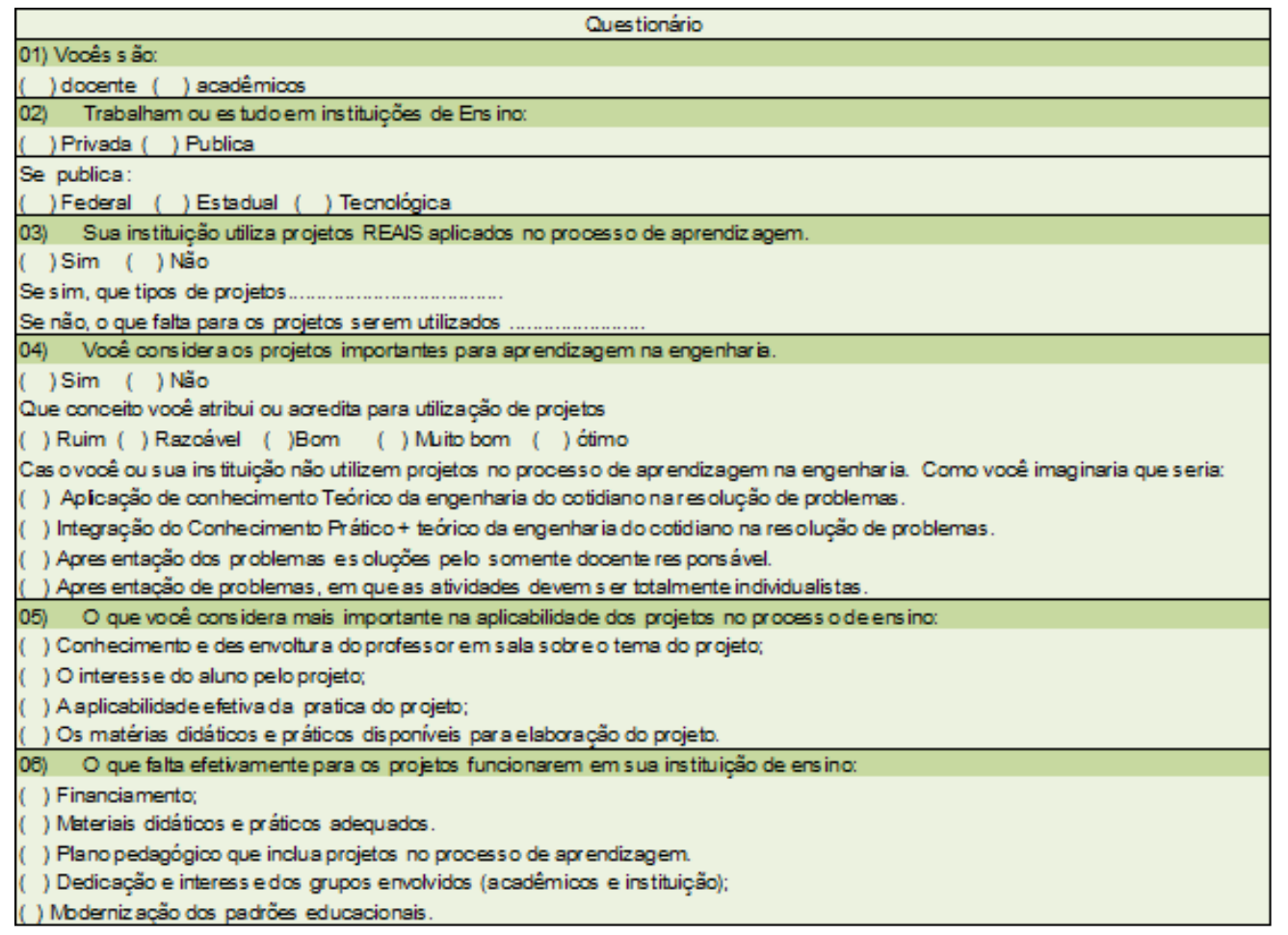

As áreas de estudo foram às cidades de Foz do Iguaçu - PR e Toledo, PR (locais onde foi realizada a pesquisa), especificamente no Centro Universitário União das Américas (Uniamérica) e Universidade Federal da Integração Latino-Americana (UNILA) situadas na cidade de Foz de Iguaçu/PR e na Universidade Tecnológica Federal do Paraná (UTFPR) campus Toledo/PR. As pesquisas foram realizadas entre os dias 03/09/2018 há 21/09/2018 paralelamente em diferentes instituições de ensino e aplicadas para docentes e professores, totalizando 42 participantes.

ALMEIDA, M.C. et al. Aprendizagem Baseada em Projetos no Curso de Engenharia Civil: na Visão de Diferentes Instituições, Docentes e Acadêmicos.
Pleiade, 14(30): 71-78, Jan.-Jun., 2020 DOI: 10.32915/pleiade.v14i30.667 
Após realizado o questionário os dados foram tabulados em tabelas no Microsoft Excel (2010) e submetidos a um teste de médias (Tukey) ao nível de 95\% significância. para confirmar diferenças entres diferentes visões (Instituições, Docentes e acadêmicos) para utilização dos projetos.

As perguntas definidas para este artigo foram baseadas na literatura e experiência dos autores sobre a utilização de projetos no processo de aprendizagem na sala de aula.

\section{RESUltados E DiscuSSÃo}

Um total 42 pessoas responderam o questionário de forma colaborativa. Destes 88,4\% são acadêmicos e 11,6\% são docentes (figura 1). Dos entrevistados 60,5 \% estudam ou trabalham em instituições de ensino privada e 39,5\% em instituições públicas (federal estadual ou tecnológicas) (figura 2).

Figura 1. Público entrevistado sobre o processo de aprendizagem através de projeto no curso de engenharia civil.

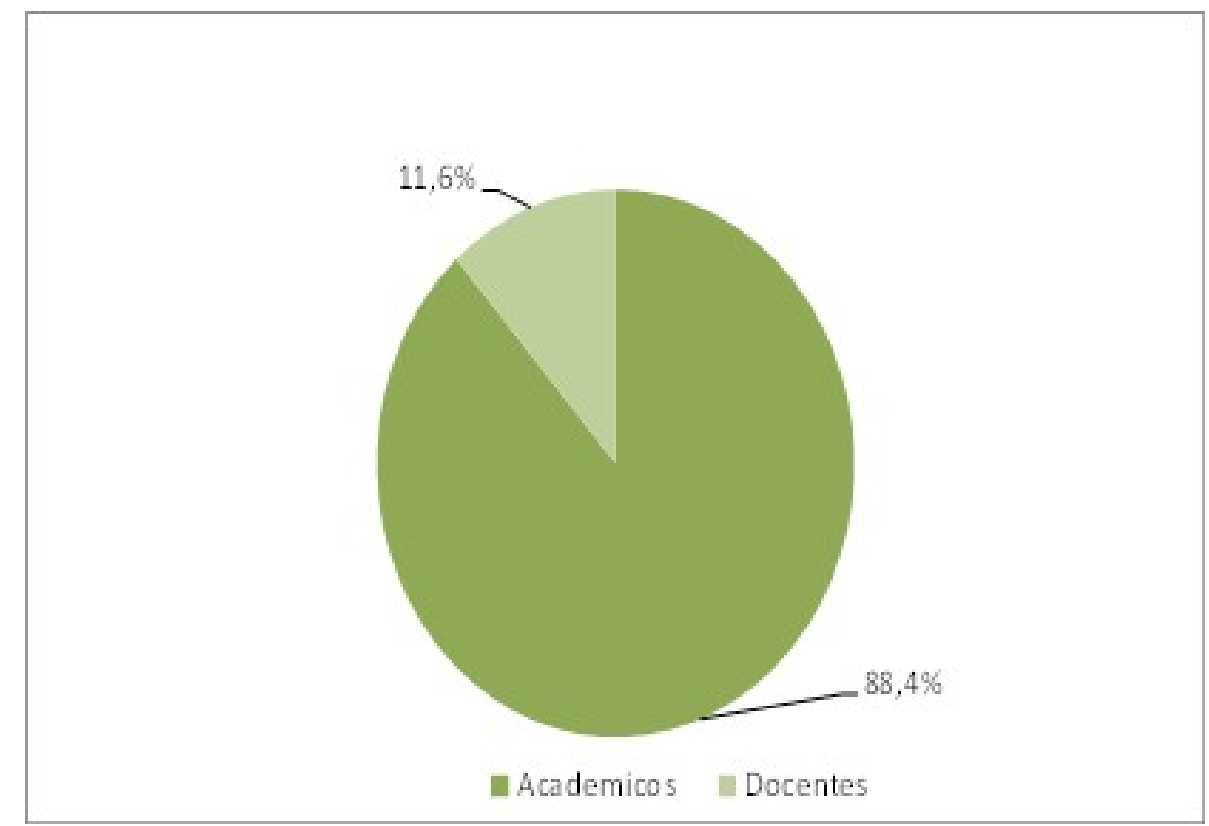

Quando questionados sobre se sua instituição utiliza projetos reais aplicados no processo de aprendizagem, $72,1 \%$ afirmaram que sim e $27,9 \%$ responderam que não (figura 3). Os que responderam sim, que os projetos são aplicados através de projetos integradores que tentam solucionar situações do cotidiano, tais como, readequação de estradas, parques, pontes moradias, cálculos relacionados à engenharia civil, projetos arquitetônicos e a construção de maquetes de pontes por exemplo. E os que não utilizam afirmaram que falta de investimento em aulas praticas e a limitação à sala de aulas seja a maior barreira a ser vencida. 
Figura 2. Público entrevistado sobre qual instituição de ensino trabalha ou estudam.

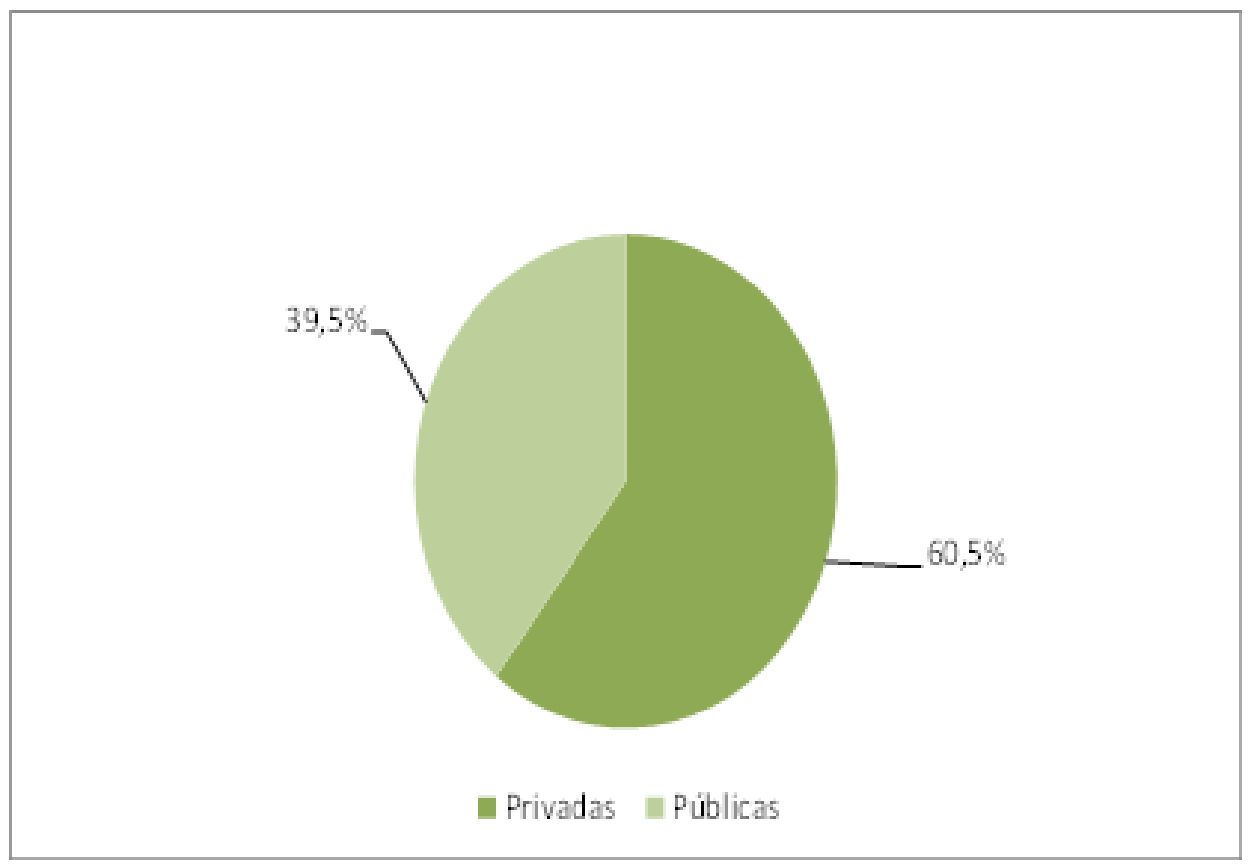

Figura 3. Público entrevistado sobre se instituição utiliza projetos reais aplicados no processo de aprendizagem.

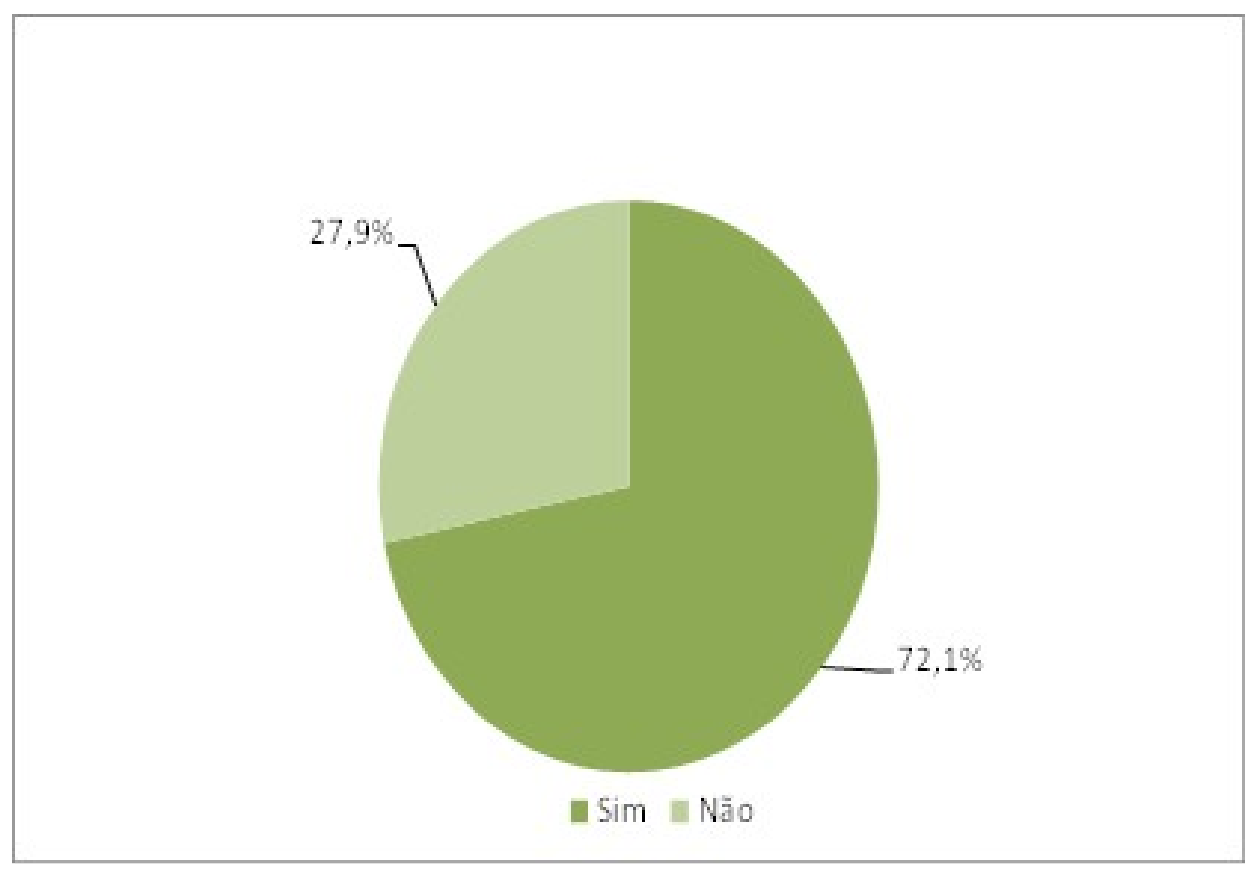

A caracterização das metodologias ativas, no Brasil, tem um componente social muito acentuado e demonstram a problematização da realidade local, como e verificado neste trabalho, pois os projetos integradores são através de problemas enfrentados regionalmente ou situações que necessitem a intervenção para soluções de problemas. Cipolla (2016) reforça que a atividade de ensino e aprendizagem deve seguir um protocolo de práticas ligadas à realidade social do país/região. 
Formar profissionais com habilidades técnicas e comportamentais necessárias é um desafio também para o docente, que precisa rever seus métodos de ensino. A metodologia de aprendizagem baseada em projetos pode ser uma opção para tornar a sala de aula mais produtiva, menos cansativa e, acima de tudo, atraente e desafiadora ao estudante (PAULA, 2017). A metodologia de aprendizagem baseada em projetos deve favorecer o desenvolvimento de um cenário prático, ao mesmo tempo em que desenvolve habilidades essenciais de um profissional bem preparado.

Todos entrevistados (100\%) consideram os projetos importantes para aprendizagem na engenharia e $53,5 \%$ dos atribuem o conceito muito bom para utilização de projeto e 30,2\% consideram ótimo, 14\% bom e 2,3 \% ruim (Figura 4). Quando estes dados são agrupados em aspetos positivos (muito bom, ótimo e bom) e aspectos negativos (ruim) e testado estaticamente a 95\% de significância é possível verificar uma diferença significativa entre os aspectos testados em que caracteres positivos diferenciam se dos negativos de forma acentuada.

Figura 4. Público entrevistado qual conceito atribuem para a utilização de projetos no processo de ensino.

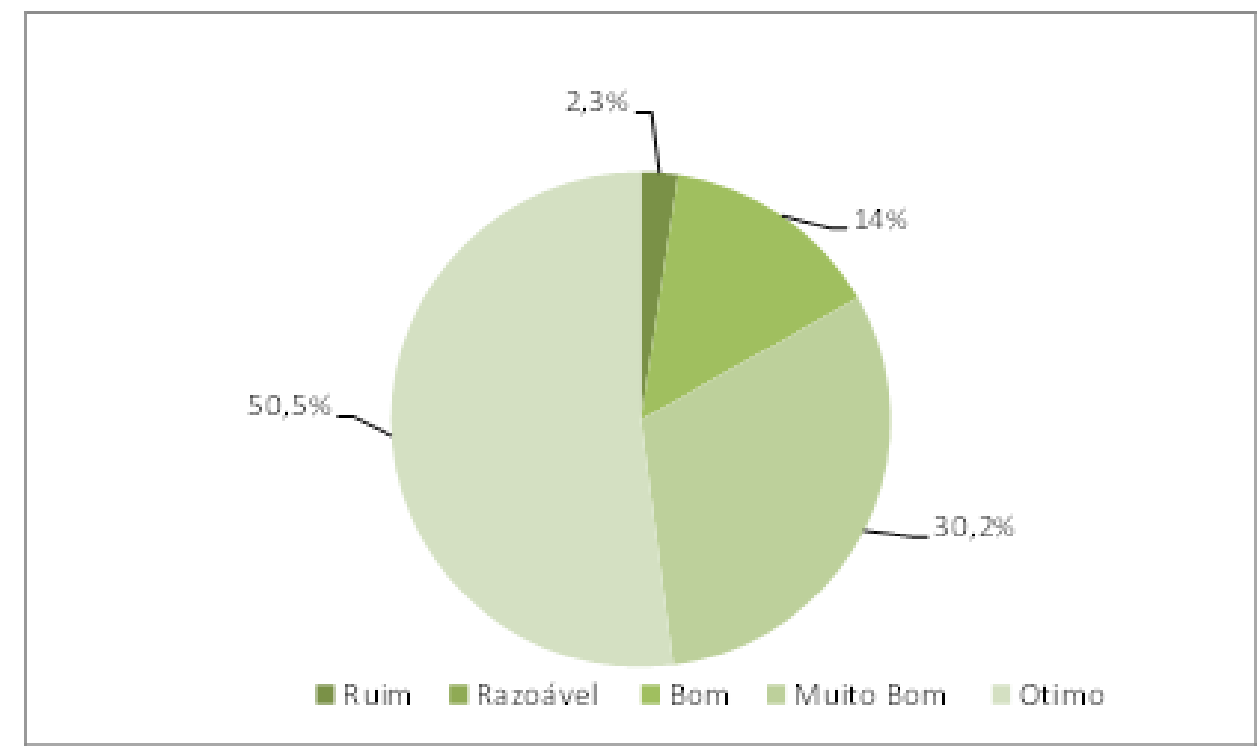

Para as pessoas questionas que não utilizam projetos no processo de aprendizagem na engenharia (68,6\%) acreditam que os projetos devem abordar Integração do conhecimento Prático + teórico da engenharia do cotidiano na resolução de problemas, e 28,6\% aplicação de conhecimento Teórico da engenharia do cotidiano na resolução de problemas. E quando perguntando sobre o que consideram mais importante na aplicabilidade dos projetos no processo de ensino, 46,5\% das pessoas consideram que projeto deve ser aplicado de forma efetiva na prática, 23,3\% acreditam ser mais importante o material didático e prático disponível para elaboração do projeto. E segundo os entrevistados que falta efetivamente para os projetos funcionarem em sua instituição de ensino é o maior interesse das partes envolvidas.

Segundo Campos (2011), a Aprendizagem Baseada em Projetos (PBL) tem sido um dos principais focos da discussão não apenas como abordagem de aprendizagem ativa, mas como alternativa para se elaborar currículos e se adotar práticas inovadoras na educação em engenharia. PBL é uma estratégia de ensino e aprendizagem do século XXI, que passa a exigir muito mais empenho dos alunos e dos professores. 
Com os resultados é possível inferir quanto os projetos podem contribuir para formação profissional dos acadêmicos do curso de engenharia, pois em seu futuro projeto é possível encontrar uma situação semelhante ao estudado no projeto. Santin \& Ahlert (2017) demonstram que na vida profissional futura o estudante encontrará o mesmo cenário, onde deverá assumir responsabilidades frente aos desafios e também os riscos inerentes às suas decisões. De esta forma os projetos demonstram ser um aliado a formação dos futuros engenheiros.

O desenvolvimento de um Projeto de Engenharia é um processo complexo, pois envolve a aptidão dos alunos para o desenvolvimento dos mesmos considerando todas as variáveis envolvidas, como a limitação do tempo e dos recursos, entre outros, bem como as competências e habilidades adquiridas; a reorganização do espaço da sala de aula para facilitação da interação e do desenvolvimento compartilhado do conhecimento; integração das novas tecnologias computacionais; reavaliação do sistema de avaliação, entre outras (MASSON et al., 2012).

Estas são sugestões que contribuiriam para tornar os projetos mais atrativos para os grupos envolvidos e efetivar a prática do projeto. O professor precisa atuar como mentor entre os problemas que os alunos enfrentarão e a prática desenvolvida e os alunos devem adquirir habilidades para resolução de problemas e desenvolver um produto final. Ambos os grupos precisam entender seu papel neste processo de aprendizagem o professor com suas orientações e os alunos de desenvolver as atividades propostas.

Apresentar ao estudante que existe ligação entre a teoria da sala de aula e a prática do mercado de traba lho é um marco importante para despertar nele o interesse pelo assunto, afinal, quando é visível a utilidade do que se está aprendendo e se espera um futuro profissional na área, a dedicação passa a ter uma recompen sa. Esse é um dos resultados alcançados com a utilização da metodologia de aprendizagem baseada em projetos (Santin \& Ahlert 2017).

\section{CONSIDERAÇÕES FINAIS}

Fica evidenciado que os processos de aprendizagem baseados em projetos no curso de engenharia civil contribuem para formação profissional do futuro, já que o mesmo enfrentará situações cotidianas ao estudado no projeto. Para eficácia dos projetos é necessário interesse de ambos os grupos envolvidos (docentes e acadêmicos) e cada um desenvolva o seu papel dentro do contexto.

Essa nova metodologia de ensino vem como uma modernização e avanço educacional ao ensino tradicional principalmente em utilizado universidades pública. E deve ser uma ferramenta adicional para formação do acadêmico em seu curso de engenharia, desenvolvendo suas habilidades para resolução de problemas.

A utilização de projetos com situações reais tornam as aulas mais atrativas e práticas, pois os acadêmi cos têm plena consciência que enfrentaram situações reais já vistas no período acadêmico.

\section{REFERÊNCIAS}

ARAÚJO, U. F. Temas Transversais e a estratégia de projetos. 8. ed. São Paulo: Moderna, 2008.

CAMPOS, L.C., Aprendizagem Baseada em projetos: uma nova abordagem para a Educação em Engenharia. In: COBENGE, 2011. Blumenau, Santa Catarina: S. E., 2011.

ALMEIDA, M.C. et al. Aprendizagem Baseada em Projetos no Curso de Engenharia Civil: na Visão de Diferentes Instituições, Docentes e Acadêmicos.
Pleiade, 14(30): 71-78, Jan.-Jun., 2020

DOI: $10.32915 /$ pleiade.v14i30.667 
CIPOLLA, L. E. Aprendizagem baseada em projetos: a educação diferenciada para o século XXI. 17v. n(3). Cientifica. Rio de Janeiro-RJ. 2016. 19p.

MASSON, T. J et al. Metodologia de ensino: aprendizagem baseada em projetos (PBL). In: CONBEGE - Congresso Brasileiro de Engenharia. 2012. Belém-Pará. 10p.

PAULA, R.V Aprendizagem baseada em projetos: Estudo de caso em um curso de Engenharia de Produção. Itajubá, jan. 2017. Disponível em: <https://repositorio.unifei.edu.br/xmlui/bitstream/handle/123456789/679/dissertacao_paula_2017.pdf?sequence=1>. Acesso em: 28/10/2018.

SANTIN, G. C. \& AHLERT, M. E. Aplicação da metodologia de aprendizagem baseada em projetos em curso de educação profissional. Lajeados - RS. UNIVATES. 2017. 20p.

TOYOHARA, Q. K. et al. Aprendizagem Baseada em Projetos: uma nova Estratégia de Ensino para o Desenvolvimento de Projetos . CEETEPS/UNESP. São Paulo - SP. 2010. 8-12p. 